\title{
La concesión del puerto de Manta y su incidencia en el desarrollo de la provincia de Manabí.
}

Manta port concession and its influence on Manabí province development.

\author{
Carlos Xavier Oleas Lara. ${ }^{1}$ Juan Pablo Palaguachi Sumba. ${ }^{2}$, Guido Javier Mazón Fierro. ${ }^{3}$ \\ \& Xavier Alejandro Guerra Sarche. ${ }^{4}$
}

Recibido: 10-03-2019 / Revisado: 15-03-209 /Aceptado: 04-04-2019/ Publicado: 13-05-2019

\begin{abstract}
.
DOI: https://doi.org/10.33262/cienciadigital.v3i2.455

The origin of ports arises worldwide as a result of the primary human being necessity of placing their settlements close to the ocean or rivers as strategic points to obtain food and communication means. These necessities along with rational and intellectual human development, led to the construction of the first boats which made easier move far away from the coast and reach new regions, stablishing in this way the maritime transportation mode. In this context, Ecuador has four commercial ports being Manta deep-water port, one of the main ones inside the country. Considering this fact, the main objective of this paper is to determine whether the concession of this port will benefit or not the commercial and economic development of the Manabí province where this port is located. In order to achieve this, aim a descriptive, inductive, and observation-based research methodology was applied, using administrative data from 2015, when the concession was not introduced yet. The results of the investigation revealed an imperative necessity of integrate different social segments into the concession process. In conclusion, it is clear a lack of road complementary infrastructure which must be considered as a priority for the authorities involved into the Manta deep-water concession.
\end{abstract}

${ }^{1}$ Escuela Superior Politécnica del Chimborazo, Facultad de Administración de Empresas, Carrera de Gestión del Transporte, Riobamba, Ecuador, carlos.oleas@espoch.edu.ec

${ }^{2}$ Escuela Superior Politécnica del Chimborazo, Facultad de Administración de Empresas, Carrera de Gestión del Transporte, Riobamba, Ecuador, juan.palaguachi@espoch.edu.ec

${ }^{3}$ Escuela Superior Politécnica del Chimborazo, Facultad de Administración de Empresas, Carrera de Gestión del Transporte, Riobamba, Ecuador, guido.mazon@espoch.edu.ec

${ }^{4}$ Escuela Superior Politécnica del Chimborazo, Facultad de Administración de Empresas, Carrera de Gestión del Transporte, Riobamba, Ecuador, xavier.guerra@espoch.edu.ec 
Keywords: Concession, Port, Manta, Development, Manabí.

\section{Resumen.}

La necesidad inicial del ser humano de ubicar sus asentamientos cerca del mar como fuente de alimentación y vía de comunicación dio como resultado el origen de los puertos. Esta necesidad, conjuntamente con el desarrollo de su capacidad racional e intelectual, permitió la construcción de las primeras embarcaciones que facilitaron inicialmente alejarse de las costas y con el paso del tiempo llegar a otras regiones, lo que permitió el surgimiento del transporte marítimo. En este contexto, en el Ecuador existen alrededor de 4 puertos comerciales, siendo uno de los más importantes el Puerto de Aguas Profundas de Manta; por lo que, el objetivo de este estudio fue conocer si, la licitación del puerto de Manta favorecerá al desarrollo comercial y económico de la provincia de Manabí, partiendo de datos levantados antes de dicho proceso; para lo cual se utilizó la metodología de investigación descriptiva, inductivo y mediante la observación que permitieron obtener datos en el año 2015 de la realidad administrativa de dicho puerto; dando como resultado principal la necesidad de integrar a los actores sociales involucrados en este proceso de concesión, se puede concluir que las autoridades correspondientes deben priorizar la construcción de la infraestructura vial complementaria para la adecuada utilización del puerto de aguas profundas de Manta.

Palabras claves: Concesión, Puerto, Manta, Desarrollo, Manabí.

\section{Introducción.}

La presencia de un puerto marítimo en un país beneficia la existencia de una vía de comunicación con otras partes del mundo, donde realmente constituye un privilegio para aquellos países que cuentan con salida al mar; porque además, representa una posibilidad de comercio y desarrollo económico, no sólo para la exportación de sus propias mercancías e importaciones en función de sus necesidades de desarrollo; sino también, como una vía que puede ser utilizada por regiones y países que no cuentan con un recurso de este tipo.

Por tales razones, contar con puertos de manera general constituye una oportunidad que genera una cadena de beneficios, como el transporte para el desarrollo de la economía interna, a partir de contenedores de mercancías que deben ser distribuidos a diferentes partes y territorios, que, por razones de distancia y capacidad de carga, generan trabajo y riqueza a las poblaciones que habitan en el lugar o cerca del mismo.

La humanidad históricamente iba realizando sus asentamientos y en esta misma medida buscaba siempre la proximidad a vías marítimas o fluviales, donde finalmente tuvieran salida al mar; primero porque era una fuente de alimentación segura y luego con el 
desarrollo y presencia de embarcaciones, tener la disponibilidad de una vía de comunicación que le permitiera explorar más allá de sus límites y buscar otros lugares para poblar o encontrar aquellos recursos que deseaban.

De esa manera, los puertos constituidos históricamente, fueron desarrollándose paulatinamente de manera proporcional al desarrollo de las diferentes formaciones económicas sociales, respondiendo a las exigencias del progreso mercantil e industrial.

En la actualidad todo esto ha cambiado, porque cada país cuenta con sus fronteras y sus puertos históricos localizados con rutas marítimas determinadas; por lo tanto, la denominación y establecimiento de nuevos puertos con fines comerciales, responde a toda una serie de requerimientos que están relacionados con grandes inversiones, que deben ser realizadas para acondicionar a las exigencias de los buques de gran calado y toda una infraestructura que debe garantizar las múltiples operaciones que se deben realizar "en situ".

Por otra parte, hay que establecer grandes depósitos de contenedores y almacenes, así como vías de comunicación terrestre funcionales y en perfecto estado, que garanticen la transportación de las mercancías hasta su destino final.

Por todo lo expuesto anteriormente es de considerar lógico que se deben tomar en cuenta en este proyecto todos los estudios de factibilidad, para conocer en base a las oportunidades, el grado de riesgo presente y de avance, antes de comprometer la inversión, sin dejar a un lado la necesidad de conocer la responsabilidad de las personas que tomarán las decisiones, la actitud o predisposición de las mismas con relación a la construcción de una obra de esa naturaleza.

Teniendo en cuenta todo lo expresado con anterioridad es interés de esta investigación es conocer la factibilidad de la licitación de las aguas profundas del Puerto de Manta, considerando también la opinión de las personas que forman parte de este proyecto.

\section{Metodología.}

Para efecto de la presente investigación se toma como base fundamental investigar el beneficio del que pueda ser objeto la población de la provincia de Manabí principalmente, tomando en cuenta lógicamente los beneficios vinculados al territorio ecuatoriano.

En el presente trabajo de investigación se plantea el método científico, como el procedimiento para vincular a la investigación con la realidad en producción de conocimiento.

El método científico minimizará la influencia de la subjetividad en este trabajo de investigación empleando instrumentos para acceder al conocimiento, como es el caso de las encuestas diseñadas para ser utilizadas de acuerdo al escenario, que durante todos los 
últimos meses se ha presentado con franco hermetismo por parte de trabajadores y funcionarios.

Esta investigación por sus objetivos está clasificada como una investigación de campo, por cuanto se realizó en el mismo lugar donde se producen los acontecimientos, que es el Puerto Marítimo Manta.

La forma de esta investigación es descriptiva, por el análisis en que se desarrolla la licitación del puerto de Manta, describiendo el hecho tal cual aparece en la realidad.

Por las fuentes de consulta es bibliográfica, por cuanto se fundamenta en libros, folletos y demás escritos del proceso de licitación como referentes de la temática para que fuera pertinente la investigación.

Se utiliza el método de la observación, durante todo el proceso de investigación, mediante la guía de observación, como recurso para realizar la misma. Además, se utilizó el método inductivo, que permite la observación de los hechos para su registro la clasificación y la derivación inductiva que parte de los hechos en particular para llegar a una generalización.

Tabla 1. Operacionalización de variables.

\begin{tabular}{|c|c|c|c|c|}
\hline Variable & Descripción & Dimensión & Indicador & Instrumento \\
\hline Independiente & $\begin{array}{l}\text { Licitación del puerto } \\
\text { de manta }\end{array}$ & $\begin{array}{l}\text { Levantamiento, } \\
\text { procesamiento, } \\
\text { análisis e } \\
\text { interpretación de } \\
\text { datos. }\end{array}$ & 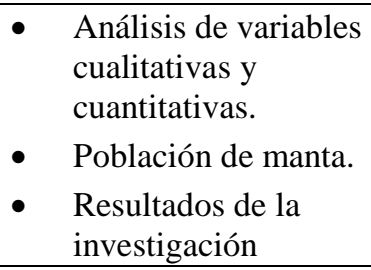 & Encuesta \\
\hline Dependiente & $\begin{array}{l}\text { Desarrollo económico } \\
\text { y comercial de la } \\
\text { Provincia de Manabí }\end{array}$ & $\begin{array}{l}\text { Investigación social } \\
\text { y administrativa. }\end{array}$ & $\begin{array}{ll}\text { - } & \text { Estudios realizados } \\
\text { - } & \text { Análisis de datos. } \\
\text { - } & \text { Procesamiento } \\
\text { informático de la } \\
\text { información }\end{array}$ & Encuesta \\
\hline
\end{tabular}

\section{Fuente: Elaboración propia}

\section{Resultados.}

El levantamiento de información, mediante la aplicación de encuestas, permitió obtener los siguientes resultados:

Pregunta 1. Considera que la información brindada a la población de Manabí con relación a la licitación del Puerto de Manta ha sido excelente, muy buena, buena, regular, deficiente.

Análisis: El 40\% de la población de Manabí consideran que la información brindada sobre la licitación del puerto de Manta ha sido regular, el 34\% la considera excelente, el $20 \%$ de los 
manabitas la valoran como buena y finalmente el $4 \%$ y $2 \%$ la consideran excelente y muy buena, respectivamente (Gráfico 1).

Información sobre la licitación del Puerto

de Manta a la población de Manabí

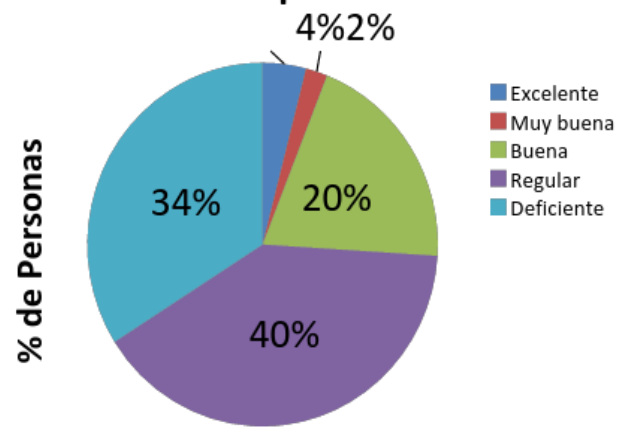

Gráfico 1. Información sobre la licitación del Puerto de Manta a la población de Manabí.

Fuente: Elaboración propia.

Interpretación: Más del 50\% de los habitantes de Manabí están inconformes con la información brindada a la población, esto puede deberse a que la información dada, no consigue esclarecer dudas y no se han planteado cuestiones que pueden ser de interés social, puesto que las decisiones que se tomen conforme avanza el proceso de licitación del Puerto de Manabí, afectará a los habitantes de la provincia.

Pregunta 2. ¿Se ha tomado en cuenta la opinión de la población en cuanto al proceso de licitación?

Análisis: El 80\% de los encuestados consideran que no se ha tomado en cuenta la opinión de la población en cuanto al proceso de licitación, mientras que el $18 \%$ opina lo contrario, el otro $2 \%$ restante de los encuestados no dan su opinión (Gráfico 2).

\section{Influye la opinión de la población sobre el proceso de licitación}

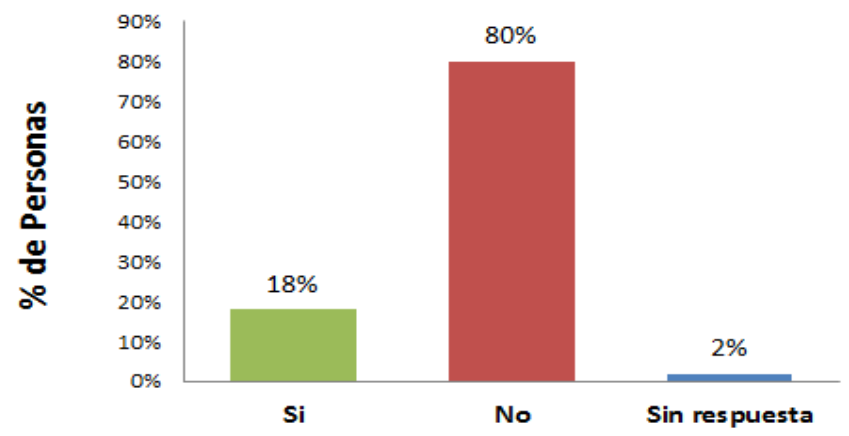

Gráfico 2. Opinión de la población sobre el proceso de licitación.

Fuente: Elaboración propia. 
Interpretación: Que exista el 18\% de población que indica, que han sido tomados en cuenta en el proceso de licitación en cuanto a su opinión es de mucha valía, puesto que las opiniones para toma de decisiones nunca van más allá de las personas con criterio formado y conocimiento de causa.

Pregunta 3. En su opinión, el proceso de licitación hasta el momento ha sido un éxito, fracaso, desconozco, sin respuesta.

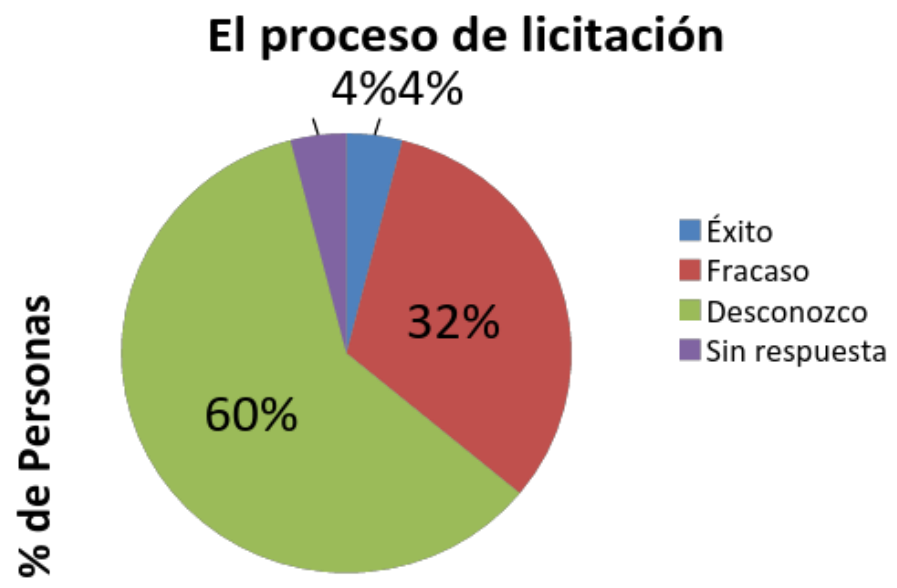

Gráfico 3. Proceso de licitación.

Fuente: Elaboración propia.

Análisis: El proceso de licitación ha sido un fracaso según la opinión del 32\% de los manabitas encuestados, un $4 \%$ de los encuestados califican al proceso de licitación como un éxito. El $60 \%$ de los encuestados afirman desconocer sobre el tema y el $4 \%$ restante no da ninguna respuesta (Gráfico 3).

Interpretación: Es de considerar que ha existido falta de información sobre el proceso de licitación, especialmente en lo que se refiere a los términos de cierre de los acuerdos alcanzado en la licitación para relacionar con los potenciales beneficios como resultado de la licitación.

Pregunta 4. ¿Ha tenido usted alguna participación en la licitación del Puerto de Manta?

Análisis: El 98\% de la población no ha participado en la licitación del puerto de Manta, y el 2\% si ha participado (Gráfico 4). 


\section{Participación en la licitación del Puerto de Manta}

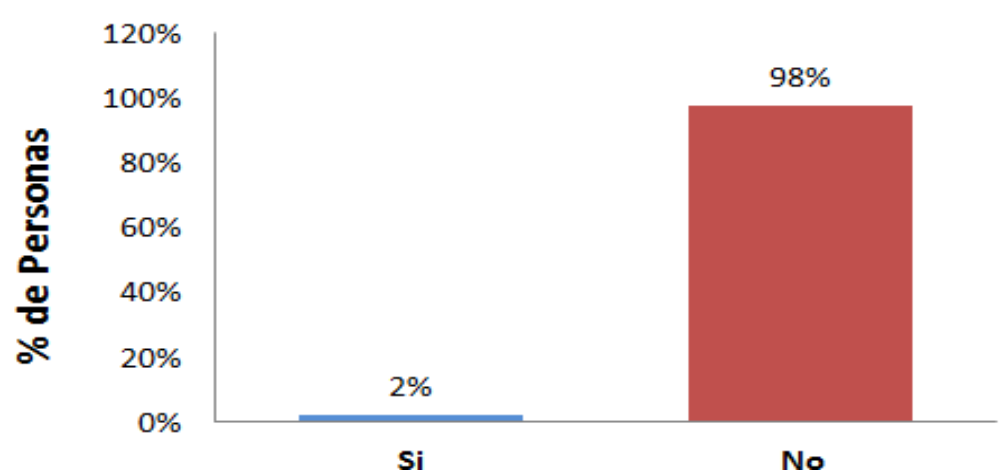

Gráfico 4. Participación en la licitación del Puerto de Manta.

Fuente: Elaboración propia.

Interpretación: al existir el 2\% de población participante de la licitación, significa que la misma fue realizada dentro de un círculo de planificadores y decidores de la licitación, cuidando que la participación se expanda. La población tomada como nuestra en relación a esta pregunta supo manifestar también; que en nada hubieran entorpecido o nada se hubiese perdido, si de vez en cuando pedían la opinión ciudadana como ahora (refiriéndose a nuestra encuesta), para sentir que de algo participaban.

Pregunta 5. La información a la población los trabajadores del puerto acerca del estado en que se encuentra el proceso de licitación es, suficiente, insuficiente, nula, sin respuesta.

Análisis: El 48\% de la población de Manabí considera nula la información a la población sobre el proceso de licitación, el $44 \%$ de manabitas la valora como insuficiente, finalmente el $6 \%$ y el $2 \%$ de los manabitas respondieron a la pregunta como nula y no dieron respuesta, respectivamente (Gráfico 5).

Información acerca del estado del proceso de licitación

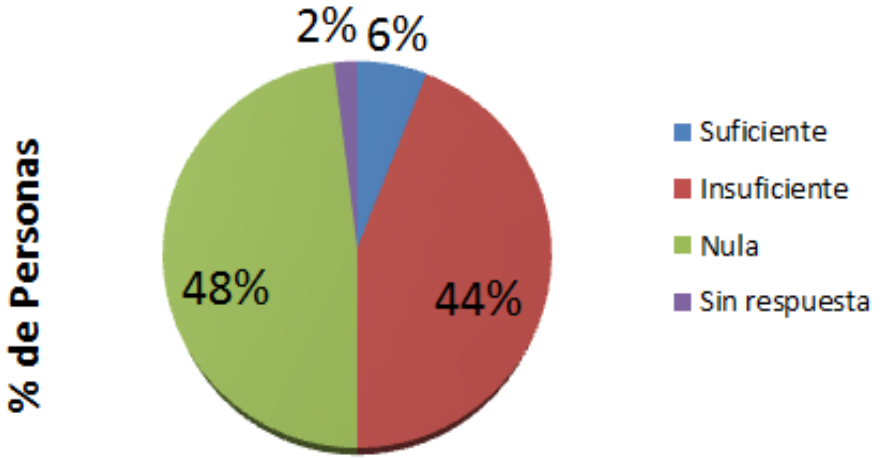

Gráfico 5. Información acerca del estado del proceso de licitación. Fuente: Elaboración propia. 
Interpretación: según los porcentajes y todo con tendencia negativa tiene una conclusión de casi el $100 \%$ de haber sido la información prácticamente nula.

Pregunta 6. ¿Considera beneficiosa la construcción del Puerto de Aguas Profundas en Manta?

Análisis: El 72\% de los manabitas consideran beneficiosa la construcción del puerto de aguas profundas en Manta; no así, el 20\% que opinó no haber sido beneficiosa la construcción de la obra y el $8 \%$ restante no dio una respuesta alguna sobre el tema (Gráfica 6).

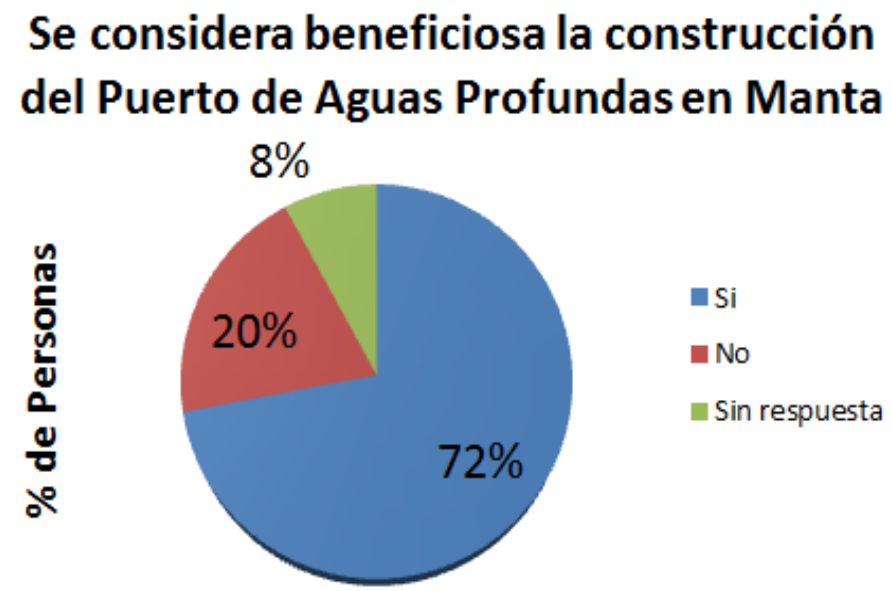

Gráfico 6. Se considera beneficiosa la construcción del Puerto de Aguas Profundas en Manta. Fuente: Provincia de Manabí

Interpretación: la mayor parte de la población de la muestra se manifestó conforme, indicando que la construcción del puerto de aguas profundas es de mucho beneficio para todos, mencionando que lo que llega al puerto de Manta no se queda en la ciudad o en la provincia; sino que va hacia el interior del país para abastecer de muchas cosas, materiales y productos que sirven para la construcción, producción y comercialización, de lo que se benefician muchos hogares por las fuentes de trabajo que esto genera normalmente en cualquier parte del mundo.

Pregunta 7. A su parecer, por la gestión realizada hasta el momento para la construcción del puerto de aguas profundas la población de Manta se siente, satisfecha, insatisfecha, sin respuesta.

Análisis: El 68\% de la población manabita está insatisfecha ante la gestión para la construcción del Puerto de Aguas Profundas, el 16\% está satisfecho y el 16\% restante no da ninguna respuesta sobre el tema (Gráfico 7). 


\section{Indice de satisfacción de los manabitas sobre la gestión para la construcción del Puerto de Aguas Profundas}

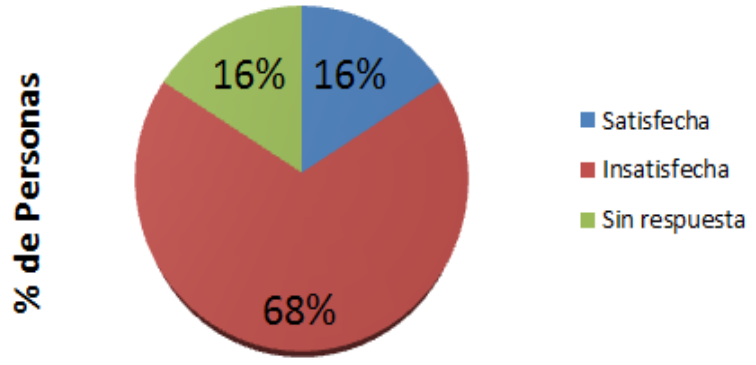

Gráfico 7. Índice de satisfacción sobre la gestión de construcción del puerto de aguas profundas. Fuente: Provincia de Manabí

Interpretación: Más de la mitad de la población se encuentra insatisfecha por la gestión para la construcción del puerto de aguas profundas en la ciudad de manta y se denota que el motivo de su insatisfacción ha sido principalmente la falta de una adecuada información permanente de los avances en cada una de las áreas de la planificación para la gestión de la construcción.

\section{Conclusiones.}

- Los resultados investigativos demuestran un alto grado de insatisfacción en la población de Manabí a consecuencia de la insuficiente información recibida acerca del proceso que se ha desarrollado en torno a la licitación del Puerto de Manta, y que se constata en un desconocimiento sobre aspectos de carácter general.

- La población no ha tenido participación, ni han sido tomadas en cuenta sus opiniones sobre la licitación del Puerto de Manta, quedando esto limitado a las personas implicadas en ese proceso directamente, lo cual desde el punto de vista estratégico constituye una debilidad al no aprovechar el apoyo de la opinión pública a favor de la concreción del acuerdo por parte de los involucrados.

- La construcción del Puerto de Manta cuenta con la aceptación de la mayoría de la población, que de manera objetiva aprecia todas las oportunidades que se derivan de su funcionamiento futuro, sin embargo, no aprueban el nivel de gestión de las autoridades encargadas de su ejecución.

- Las autoridades locales, identificadas con las necesidades de desarrollo de la Provincia y del País por la construcción de un puerto de aguas profundas en Manta precisa de mayor apoyo desde el nivel estatal para poder impulsar un conjunto de obras que garantizaran un mejor funcionamiento de las operaciones portuarias, asî como el enlace necesario con la economía interna de todo el territorio nacional.

- Un apoyo popular consciente y dirigido es percibido por este trabajo, como una necesidad para lograr la preparación de la población en función del impacto que generara una obra colosal de ese tipo para que pueda favorecer un desarrollo armónico. 
- Existe predisposición negativa y errónea por parte de la muestra estudiada en cuanto al proceso de licitación de Puerto de Manta por falta de conocimiento acerca de las bondades que esta obra aporta el desarrollo de la provincia y el país.

\section{Referencias bibliográficas.}

Ascencio, L., González-Ramírez, R., \& Cedillo, G. (12 de Marzo de 2012). Comunidades logístico-portuarias. Recuperado el 21 de Febrero de 2016, de http://www.logisticamx.enfasis.com/articulos/63109-comunidades-logisticoportuarias

Autoridad Portuaria de Manta. (Noviembre de 2013). Convocatoria. Concurso Público Internacional para la Concesión de Terminales de Contenedores y Multipropósitos del Puerto de Aguas Profundas de Manta. Recuperado el 5 de Enero de 2016, de http://www.puertodemanta.gob.ec/concesionmanta

Autoridad Portuaria de Manta. (Noviembre. de 2013). Preámbulo.Concurso Público Internacional para la Concesión de las Terminales de Contenedores y Multipropósito del Puerto de Aguas Profundas de Manta. Recuperado el 5 de Enero de 2016, de http://www.puertodemanta.gob.ec/concesionmanta

Autoridad Portuaria de Manta. (2016). Antecedentes. Recuperado el 23 de Febrero de 2016, de http://www.puertodemanta.gob.ec/quienes-somos/antecedentes

Autoridad Portuaria de Manta. (2016). Misión y Visión. Recuperado el 23 de Febrero de 2016, de http://www.puertodemanta.gob.ec/quienes-somos/mision-y-vision-2

Cantos, J., Semiglia, S., \& Vera, S. (2009). Análisis del impacto económico de la concesión del Puerto de Manta con respecto al comercio exterior ecuatoriano.

CEPE-ONU. (2012). Sistemas de Comunidad Portuaria. Recuperado el 21 de Febrero de 2016, de http://tfig.unece.org/SP/contents/port-community-systems.htm

Cornejo, P. (9 de Enero de 2013). LA TOMADURA DE PELO MÁS BURLONA DE LA HISTORIA. Recuperado el 25 de Febrero de 2016, de ttps://esla.facebook.com/lafarsadelarevolucion/posts/408345019248416

Delgado, C. (22 de Noviembre de 2015). El Diario. Manabita de libre pensamiento.

EL BANCO MUNDIAL. (2016). Tráfico marítimo de contenedores (TEU: unidades equivalentes a 20 pies). Recuperado el 23 de Febrero de 2016, de http://datos.bancomundial.org/indicador/IS.SHP.GOOD.TU 
El Diario. Manabita libre de pensamiento. (26 de Julio de 2015). El puerto, una historia fallida.

Fernández, L. (10 de Octubre de 2014). Análisis de la realidad del Puerto de Manta.http://www.ccm.org.ec/1464-analisis-de-la-realidad-del-puerto-demanta.html. Cámara de Comercio de Manta.

Microsoft Corporation. (2009). Puertos (Geografía). Clasificación y tipos de puertos.

Rojas, J., \& Montero, L. (2010-). Tendencias portuarias. Recuperado el 11 de Febrero de 2016, de http://www.alv-logistica.org/docs/2JOSEROJAStendenciasPortuarias.pdf

Rúa, C. (2006). Los puertos en el transporte marítino. Recuperado el 12 de Febrero de 2016, de http://upcommons.upc.edu/bitstream/handle/2117/289/8.\%20Rua.pdf

Therán, D. (5 de Mayo de 2015). Puertos. Recuperado el 11 de Febrero de 2016, de https://prezi.com/wo2vgnsb6-wc/puertos/

UPC- Departament de Ciència i Enginyeria Nàutiques. (2004). El futuro tecnológico de las Terminales Marítimas de Vehículos: La integración de sus sistemas de información. Recuperado el 15 de Febrero de 2016, de http://www.tdx.cat/bitstream/handle/10803/7001/02Jmmc02de12.pdf;jsessionid=95 F0F512562AB269D1BBF80D39051069.tdx1? sequence $=2$ 


\section{PARA CITAR EL ARTÍCULO INDEXADO.}

Oleas Lara, C., Palaguachi Sumba, J., Mazón Fierro, G., \& Guerra Sarche, X. (2019). La concesión del puerto de Manta y su incidencia en el desarrollo de la provincia de Manabí. Ciencia Digital, 3(2), 5-16. https://doi.org/10.33262/cienciadigital.v3i2.455

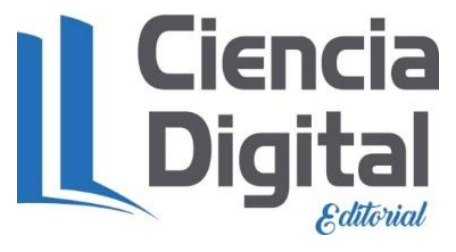

El artículo que se publica es de exclusiva responsabilidad de los autores y no necesariamente reflejan el pensamiento de la Revista Ciencia Digital.

El artículo queda en propiedad de la revista y, por tanto, su publicación parcial y/o total en otro medio tiene que ser autorizado por el director de la Revista Ciencia Digital.
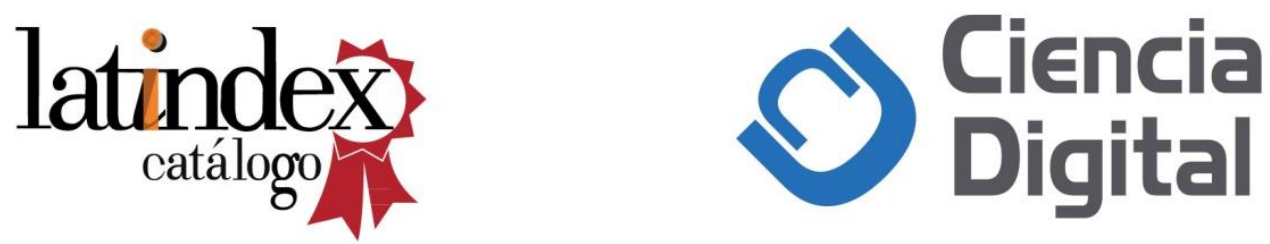